\title{
GENDER IDENTIFICATION SYSTEM FOR CRIME SCENCE ANALYSIS USING FINGERPRINTS
}

\author{
Dr. Jaison B, \\ Department of Computer Science and Engineering, \\ RMK Engineering College, \\ Kavaraipettai, Tamil Nadu, India \\ bjn.cse@rmkec.ac.in
}

\begin{abstract}
Gender identification or classification is a challenging task in computer vision as the biometrics of male and female such as fingerprints, face, vein have many variations. Among the various biometrics, fingerprints are commonly available in a crime scene. In this, study, gender identification system for crime scene analysis using fingerprints is presented. Initially, the fingerprints are de-noised by median filter and Otsu thresholding is employed to binarize the fingerprints in the preprocessing stage. Then, the features are extracted by Box-Cox transformation method. Finally, the classification is made by logistic regression classifier. A better classification accuracy of $96 \%$ is achieved by the gender identification system using Box-Cox transformation and logistic regression classifier.
\end{abstract}

Keywords: Fingerprints, gender identification, Box-Cox transformation, Logistic regression classifier.

\section{INTRODUCTION}

Multiple facial regions based gender classification using facial images is discussed in [1]. The input image is converted into gray scale. The geometric normalization is made for the image pixels. The illumination variations are reduced by histogram equalization. The classification is made by Support Vector Machine (SVM) classifier. Gender classification system using hybrid approach of geometric properties and Haar cascade classifiers is described in [2]. The input facial images are extracted by geometrical properties and Haar cascade classifiers. Illumination variations are removed by Weber illumination normalization technique. The classification is made by SVM classifier.

SVM and 3-dimentional geometrical features based gender classification is discussed in [3]. The input 3-dimentional facial images are extracted by geometrical features. Then the extracted features are classified by SVM classifier. Gender classification using iris image is discussed in [4]. At first, the preprocessing is made by Hough transform to remove localization. The features like texture and statistical features are extracted from input iris images. Then Discrete Wavelet Transform (DWT) is applied for the enhancement. The classification is made by SVM classifier.

Gender classification using fingerprints based on Gaussian Mixture Model (GMM) and wavelets is discussed in [5]. The input fingerprint images are decomposed by DWT. Then rank features are separated from detailed sub band coefficients by rank features. Then the gender classification is made by GMM. Human radiation wave analysis based gender classification is discussed in [6]. Initially, the mean frequency data is applied for the input human radiations. Then data preprocessing s made. Classification is made by k-nearest neighbor classifier.

Local Directional Pattern (LDP) based gender classification is described in [7]. The input facial images are extracted from the LDP then the face regions are 
divided into small regions and LDP is concatenated into a single vector. SVM classifier is used for classification. Gender classification for SVM learning approach for pseudo example is described in [8]. The active appearance model is applied for the input images. The texture and shape features are extracted by using statistical analysis. The histogram equalization is applied for illumination variations. The Principal Component Analysis (PCA) is used for dimensionality reduction. SVM classifier is used for classification.

Gender classification and face recognition in personal memories is presented in [9]. Firstly, the features are extracted by pose estimation technique and color. Then the normalization technique is applied. The dimensions are reduced by using PCA. The SVM classifier is used for classification. Offline handwriting images based gender classification using textural features is discussed in [10]. The Gabor filter is applied for noise removal. The standard deviation and mean features are extracted. The classification is made by using feed forward neural network.

Two-dimensional Gabor wavelet transform and SVM based gender classification is discussed in [11]. The input face images are extracted by twodimensional Gabor transform. The SVM classifier is used for the classification. Gender classification using age information is described in [12]. Initially, the features like local binary pattern, geometry and appearance features are extracted. The SVM classifier is used for gender classification.

A system to identify the gender of the suspect in a crime scene analysis using fingerprint is presented in this study. The methods and materials used for gender classification are discussed in section 2. Experimental results and discussion of gender classification system is described in section 3. The last section gives the conclusion of gender classification system using fingerprints.

\section{METHODS AND MATERIALS}

The gender identification system for crime scene analysis using fingerprints of male and female is presented. This system is built by three main stages they are pre-processing, feature extraction and classification. In preprocessing stage, the median filter is applied for input fingerprint images to remove noises and Otsu's thresholding is used for edge detection. The features are extracted by using box-Cox transformation technique. The logistic regression classifier is used for the classification. Figure 1 shows the overall work flow of gender classification system.

\section{A. Preprocessing}

Preprocessing is an important stage in gender identification system using fingerprints. Initially, the input fingerprint images are converted into grayscale image. Then, the median filter is applied to remove noise. To detect the edge information, a global thresholding approach Otsu's thresholding is applied. Otsu's thresholding is also used for brain tissue segmentation [13]. Then the edge detected image is the input to the feature extraction stage. 
Int. J.Adv.Sig.Img.Sci, Vol. 3, No.2, 2017

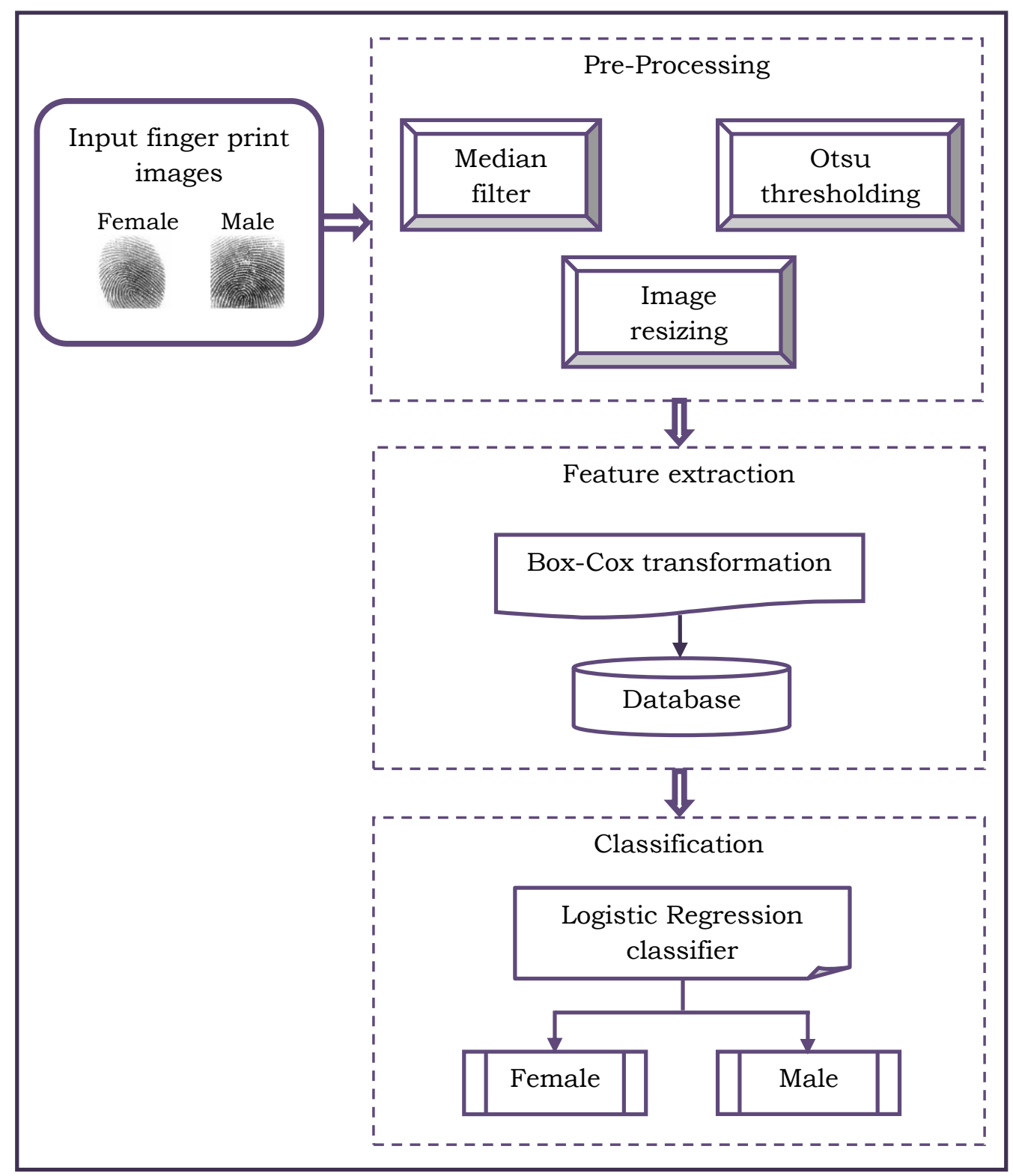

Fig. 1 Gender identification system - workflow

\section{B. Box-Cox transformation technique}

Box-Cox transformation is a valuable method for the distribution of unknown variable. The Box-Cox transformation of the variable $z$ is represented by $\omega$, and is defined by,

$$
z_{\omega}^{\prime}=\frac{z^{\omega}-1}{\omega}
$$


where $z^{\omega}$ is the turkey transformation, when $\omega<0$, both $z_{\omega}$ and $z_{\omega}^{\prime}$ may change the sign of $z^{\omega}$ to preserve the order. When $\omega=0$, then the Box-Cox equation can be rewrite as,

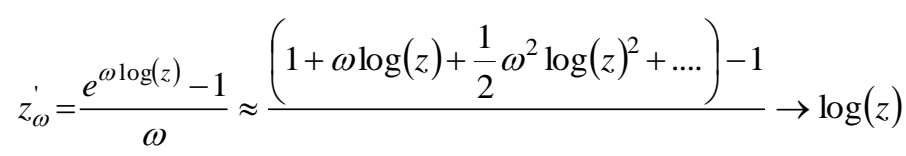

Here $z_{\omega}^{\prime}$ is $z=1$ and maps the point $z_{\omega}^{\prime}=0$ for all the values of $\omega$. The oneparameter Box-Cox transformations is given by,

$$
z_{i}^{(\omega)}= \begin{cases}\frac{z_{i}^{\omega}-1}{\omega} & \text { if } \omega \neq 0, \\ \ln z_{i} & \text { if } \omega=0\end{cases}
$$

The two-parameters of Box-Cox transformation is given by,

$$
z_{i}^{(\omega)}=\left\{\begin{array}{l}
\frac{\left(z_{i}+\omega_{2}\right)^{\omega_{1}-1}}{\omega_{1}} \text { if } \omega_{1} \neq 0, \\
\ln \left(z_{i}+\omega_{2}\right) \text { if } \omega_{1}=0,
\end{array}\right.
$$

The first transformations hold for $z_{i}>0$, and the second hold for $z_{i}>-\omega_{2}$. The parameter $\omega$ is defined by profile likelihood function. Box-Cox transformation is also used in visual encoding [14] and also in forecasting practice [15]. In this study, the Box-Cox transformation is used for extracting dominant features from fingerprints. After transformation, the extracted features are stored in the feature database which is used as the input for classification.

\section{Logistic regression classification}

Logical regression is a supervised classification algorithm. The binary dependent variable is modeled by logistic function which uses logistic regression. In logistic regression classification the target variable can take only a discrete amount of features from the given set. It is a regression model. The regression model is built by the probability of the given data. In linear regression the data follows a linear function the logistic regression model follows a sigmoid function. The logistic regression is given by,

$$
k(y)=\frac{1}{1+e^{-y}}
$$

Logistic regression performs a classification by decision threshold method. The thresholding value setting is an important aspect of logistic regression and depends on the classification problem. Logistic regression is also used in telecom customer churn prediction [16] and pedestrian detection method [17]. In this study, the logistic regression is used for the gender classification using fingerprints. 


\section{RESULTS AND DISCUSSION}

The performance of the gender identification system using fingerprints is discussed in this section. The database contains fingerprints from 80 females and 100 males with different ages. The fingerprints are collected by the scanner using the Fingkey Hamster II scanner of 500 resolution dpi. Fingerprints in database are 260x300 pixels of size. The fingerprint from each person is collected for analysis and classified individually. Figure 2 and Figure 3 shows the fingerprints collected fingerprints from the database.
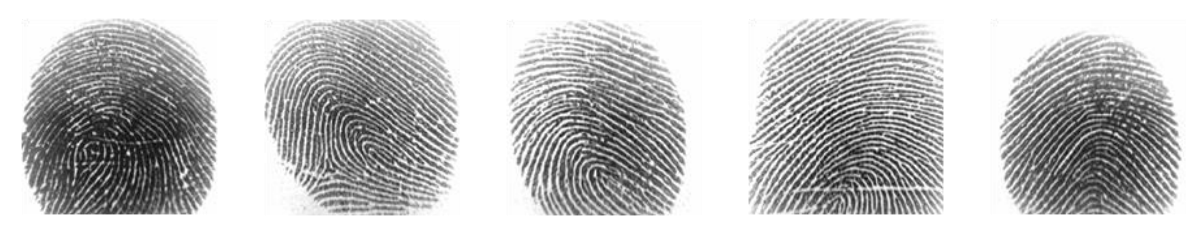

Fig. 2 Sample fingerprints in database - Female
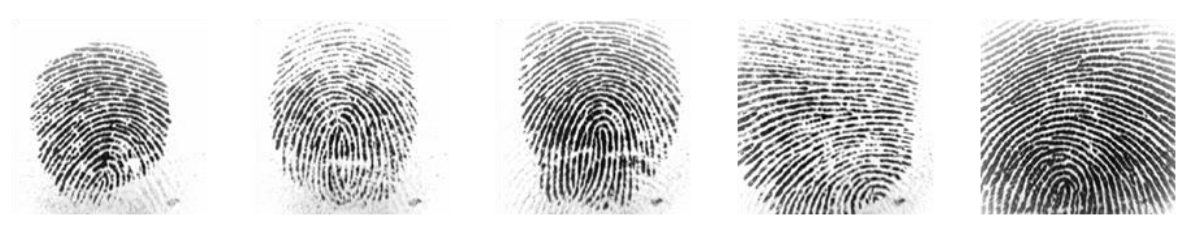

Fig. 3 Sample fingerprints in database - Male

At first, the input fingerprint images are preprocessed and edge detected by using median filter and Otsu's thresholding respectively. Then the Box-Cox transformation technique is applied to extract features. It uses $\omega$ values for transformation. Table 1 shows the classification accuracy of gender classification using fingerprints based on Box-Cox transformation and logistic regression classifier.

\section{TABLE 1 Classification accuracies based on Box-Cox transformation and} logistic regression classifier

\begin{tabular}{|c|c|c|}
\hline Input image size & $\omega$ value & Classification accuracy (\%) \\
\hline $16 \times 16$ & 2.4 & 83.50 \\
\hline $16 \times 16$ & 2.2 & 88.00 \\
\hline $16 \times 16$ & 2.0 & 90.00 \\
\hline $32 \times 32$ & 1.8 & 94.00 \\
\hline $32 \times 32$ & 1.6 & 82.00 \\
\hline $32 \times 32$ & 1.4 & 96.00 \\
\hline $64 \times 64$ & 1.2 & 95.00 \\
\hline $64 \times 64$ & 1.0 & 93.50 \\
\hline $64 \times 64$ & 0.8 & 94.00 \\
\hline $128 \times 128$ & 0.6 & 95.00 \\
\hline $128 \times 128$ & 0.4 & 90.50 \\
\hline $128 \times 128$ & 0.2 & 95.00 \\
\hline Average classification accuracy (\%) & $\mathbf{9 1 . 3 7}$ \\
\hline
\end{tabular}


For performance analysis, the fingerprint images are resized into $16 \times 16$, $32 \times 32,64 \times 64$ and $128 \times 128$. It is observed from Table 1 that an average identification rate of $91.37 \%$ is obtained by using Box-Cox transformation and logical regression classifier. The maximum classification accuracy of $96 \%$ is obtained while using $32 \times 32$ pixel sized fingerprint images and $\omega$ value of 1.4 . The minimum classification accuracy is $82 \%$ for $32 \times 32$ pixel sized fingerprint image and its $\omega$ value is 1.6 .

\section{CONCLUSION}

An efficient method for gender classification using fingerprint images is presented for crime scene analysis using Box-Cox transformation and logistic classifier. The input images are resized into four different sizes $16 \times 16,32 \times 32$, $64 \times 64$ and $128 \times 128$ pixels for performance evaluation. At first the median filter is used for noise removal, the otsu's thresholding and binarization is made for edge detection. Then, the Box-Cox transformation method is applied with the different $\omega$ values. Then these features are stored in the database and used as input for classification. Then logistic regression classifier is used for classification. Results shows the better classification accuracy of $91.37 \%$ using the Box-Cox transformation and logistic regression classifier.

\section{REFERENCES}

[1]. L. Lu, Z. Xu, and P. Shi, "Gender classification of facial images based on multiple facial regions", WRI World Congress on Computer Science and Information Engineering, Vol. 6, 2009, pp. 48-52.

[2]. P. Goel, and S. Agarwal, "Hybrid approach of haar cascade classifiers and geometrical properties of facial features applied to illumination invariant gender classification system", International Conference on Computing Sciences, 2012, pp. 132-136.

[3]. X. Han, H. Ugail, and I. Palmer, "Gender classification based on 3D face geometry features using SVM", International Conference on CyberWorlds, 2012, pp. 114-118.

[4]. A. Bansal, R. Agarwal, and R.K, Sharma, "SVM based gender classification using iris images", International Conference on Computational Intelligence and Communication Networks, 2012, pp. 425-429.

[5]. D.G. Rajesh, and M. Punithavalli, "Wavelets and Gaussian mixture model approach for gender classification using fingerprints", International Conference on Current Trends In Engineering and Technology, 2014, pp. 522-525.

[6]. S.Z.A. Jalil, M.N. Taib, H.A. Idris, and M.M. Yunus, "Gender classification based on human radiation wave analysis", International Conference on Computer Modelling and Simulation, 2011, pp. 59-63.

[7]. T. Jabid, M.H. Kabir, and O. Chae, "Gender classification using local directional pattern (LDP)", International Conference on Pattern Recognition, 2010, pp. 2162-2165.

[8]. H. Chen, and W. Wei, "Pseudo-example based iterative svm learning approach for gender classification", World Congress on Intelligent Control and Automation, 2006, Vol. 2, pp. 9528-9532. 
[9]. F. Grangeiro, R. Jesus, and N. Correia, "Face recognition and gender classification in personal memories", International Conference on Acoustics, Speech and Signal Processing, 2009, pp. 1945-1948.

[10]. A. Mirza, M. Moetesum, I. Siddiqi, and C. Djeddi, "Gender classification from offline handwriting images using textural features", International Conference on Frontiers in Handwriting Recognition, 2016, pp. 395-398.

[11]. W. Chuan-xu, L. Yun, and L. Zuo-yong, "Algorithm research of face image gender classification based on 2-d gabor wavelet transform and svm", International Symposium on Computer Science and Computational Technology, Vol. 1, 2008, pp. 312-315.

[12]. J.B. Ko, W. Lee, S.E. Choi, and J. Kim, "A gender classification method using age information", International Conference on Electronics, Information and Communications, 2014, pp. 1-2.

[13]. P. Kalavathi, "Brain tissue segmentation in MR brain images using multiple Otsu's thresholding technique", International Conference on Computer Science \& Education, 2013, pp. 639-642.

[14]. R. Maciejewski, A. Pattath, S. Ko, R. Hafen, W.S. Cleveland, and D.S. Ebert, "Automated box-cox transformations for improved visual encoding. IEEE transactions on visualization and computer graphics", Vol. 19, No. 1, 2012, pp. $130-140$.

[15]. G. Renxiang, Z. Shiying, and L. Bao, "Forecasting practice from Box-Cox transformation models", Journal of Systems Engineering and Electronics, Vol. 8, No. 3, 1997, pp. 27-33.

[16]. P. Li, S. Li, T. Bi, and Y. Liu, "Telecom customer churn prediction method based on cluster stratified sampling logistic regression', International Conference on Software Intelligence Technologies and Applications \& International Conference on Frontiers of Internet of Things 2014, pp. 282287.

[17]. J. Kim, J. Lee, C. Lee, E. Park, J. Kim, H. Kim, and H. Jeong, "Optimal feature selection for pedestrian detection based on logistic regression analysis", International Conference on Systems, Man, and Cybernetics, 2013, pp. 239-242. 